\title{
Regional Emergence of Start-Ups in Information Technologies: The Role of Knowledge, Skills and Opportunities
}

\author{
Michael Fritsch \\ Professor, Faculty of Economics and Business Administration, m.fritsch@uni-jena.de \\ Friedrich Schiller University Jena, Carl-Zeiß-Straße, 307743 Jena, Germany \\ Michael Wyrwich \\ Professor, Faculty of Economics and Business, m.wyrwich@rug.nl \\ University of Groningen, Nettelbosje 2, 9747 AE Groningen, Netherlands
}

\begin{abstract}
$\mathrm{W}$ e investigate the regional emergence of new information technology start-ups in Germany. The largest share of these start-ups is located in cities or densely populated regions that are well equipped with institutions of higher education and research. The

empirical analysis clearly indicates the critical role of industry-specific knowledge plays for new. Hence, strengthening the regional knowledge base should be a key policy that aims at stimulating entrepreneurship in this sector.
\end{abstract}

\section{Keywords:}

innovative start-ups;

information technologies; universities;

regional knowledge
Citation: Fritsch M., Wyrwich M. (2019) Regional Emergence of Start-Ups in Information Technologies:The Role of Knowledge, Skills and Opportunities. Foresight and STI Governance, vol. 13, no 2, pp. 62-71. DOI: $10.17323 / 2500-2597.2019 .2 .62 .71$ 
$\mathrm{O}$ ver the last several decades information technologies (IT) have emerged as an important sector that has a strong economic impact on the economy and on our lives. It is generally expected that the role of IT industries will become even stronger in the foreseeable future. Regions that host many viable IT firms may benefit from the growing importance of this industry in two ways. First, these firms may grow and directly create employment and wealth. Second, the regional economy may benefit in indirect ways from IT knowledge, for example, through the availability of IT skills and services.

This paper analyzes the territorial aspects of the emergence of new IT firms. Why do some regions have a relatively large number of start-ups while others have virtually none? What is the role of knowledge, skills, and agglomeration economies in the emergence of IT firms? The following sections provide an overview on the potential determinants of IT start-ups at the regional level, introduce the data and report on the spatial distribution of IT start-ups in Germany in the period 2009-2016, and present the results of the empirical analysis. The final section draws conclusions from these findings.

\section{What Determines the Regional Emergence of IT Start-ups?}

Based on the literature dealing with regional determinants of entrepreneurial activity, one can identify two groups of factors that may shape the emergence of IT start-ups: knowledge and the availability of resources. According to the knowledge spillover theory of entrepreneurship [Acs et al., 2009; 2013], the knowledge generated by incumbent firms, universities, public research institutes, and others is a key source of business ideas (entrepreneurial opportunities) that may lead to the creation of a start-up. Spatial proximity to such sources of knowledge can be important because new ideas do not flow freely across space but tend to be regionally bounded [Asheim, Gertler, 2006; Boschma, 2005]. Hence, people located close to such knowledge sources are considerably more likely to absorb and apply the relevant knowledge. Given that founders show a pronounced tendency to locate their firms in close spatial proximity to their residence [Figueiredo et al., 2002; Dahl, Sorenson, 2009], startups are most likely to emerge in close proximity with the relevant knowledge sources. The availability of re- sources includes appropriate labor, finance, and other factors that the start-ups need to survive and grow.

In our analysis, we capture the IT-specific knowledge by

- the presence of higher education institutions (HEIs). We distinguish between regular universities and applied sciences universities (Fachhochschulen). ${ }^{1}$

- the size of these departments in terms of financial budget.

- the intensity of third-party funding measured as the share of third-party funds in the total budget of the department. Since third-party funds are almost always allocated via some kind of competitive procedure, they can be regarded as an indication of research quality. ${ }^{2}$

- the regional employment shares in IT hardware and software industries.

These measures, particularly the regional employment shares in IT hardware and software, also indicate the availability of qualified personnel.

Agglomeration economies and diseconomies as measured by population density may be another important factor for IT start-ups. Agglomeration economies are made possible by large and productive labor markets where special qualifications are available, financial institutions are present, and there is a rich supply of supportive services [Helsley, Strange, 2011]. Agglomeration diseconomies emerge as a result of intense competition for resources that results in comparatively high rents and wages. The density and proximity of people increases the frequency of (face-to-face) interactions among heterogeneous actors, which provides an important basis for knowledge sharing and effective learning (e.g., [Jacobs, 1969; Helsley, Strange, 2011; Glaeser, Sacerdote, 2000; Storper, Venables, 2004].

Based on the knowledge spillover theory of entrepreneurship, we expect a positive relationship between the different measures of knowledge and the regional levels of new business formation. The estimated coefficients for these knowledge sources can be regarded as an indication of their relative importance. Is education and research at regular universities more or less important than education and research at the universities of applied sciences? Is it more the sheer presence of the HEIs, their size, or the quality of their research that is more important? We also expect a

\footnotetext{
Regular universities and universities of applied sciences are different in many respects, including purpose, scope and size, teaching, and research [Warning, 2007]. The universities of applied sciences are mainly intended to provide undergraduate education with a focus on transferring theoretical concepts and scientific methods into practical application; these universities do not grant doctoral degrees. Courses are more structured than at regular universities and classes are smaller. Universities of applied sciences tend to have a relatively strong focus on the regional economy. Hence, their partners for R\&D cooperation are primarily small and medium sized local firms. In contrast, regular universities have a stronger focus on basic research and have a much wider regional scope. Their cooperative relationships are mainly with larger firms. On average, universities of applied sciences are much smaller in terms of personnel and students than regular universities.

We have no information about the number of students and professors in computer science, which could have been a good alternative indicator.
} 
Table 1. Overview of Indicators for Regional Determinants of IT Start-up Activity

\begin{tabular}{|l|l|}
\hline \multicolumn{1}{|c|}{ Measure } & \multicolumn{1}{c|}{ Stands for ... } \\
\hline $\begin{array}{l}\text { Presence of higher education institutions (HEIs) } \\
\text { with a department of computer science } \\
\text { Size of computer science departments at local HEIs }\end{array}$ & $\begin{array}{l}\text { IT specific knowledge and entrepreneurial opportunities, as well as the } \\
\text { availability of personnel with IT-relevant skills }\end{array}$ \\
\hline Intensity of third-party funding of HEI research & Quality of regional research and knowledge \\
\hline $\begin{array}{l}\text { Employment share in IT-hardware } \\
\text { Employment share in IT-software }\end{array}$ & IT specific knowledge and availability of personnel with IT-relevant skills. \\
\hline Population density & $\begin{array}{l}\text { Availability of resources (qualified labor, finance, other services), face to face } \\
\text { contact, other agglomeration economies and diseconomies }\end{array}$ \\
\hline Source: authors. & \\
\hline
\end{tabular}

positive relationship between population density and new business formation in IT. Table 1 provides an overview of the different indicators and their interpretation.

\section{Data Description}

Our data source for new business formation is the Enterprise Panel of the Center for European Economic Research (ZEW-Mannheim). These data are based on information from the largest German credit-rating agency, Creditreform (for a more detailed description see [Bersch et al., 2014]. As with many other data sources on start-ups, these data may not be considered comprehensive given that very small start-ups, such as those that do not have any regular employees ('solo entrepreneurs') are overlooked. However, once a firm is registered, hires employees, requests a bank loan, or conducts reasonable economic activities, even solo entrepreneurs are included in this dataset and information about their activities is gathered beginning with the 'true' date the firm was established. Hence, many solo entrepreneurs are captured along with the correct business founding date. This information is limited to the set-up of a firm's headquarters and does not include the establishment of branches. In our empirical analysis we use these data for the years 2009-2016.

Based on these start-up data, we distinguish between all IT start-ups and the sub groups (1) start-ups in software, hardware \& consulting and (2) start-ups in IT retail and leasing. The former group can be further separated into (1a) software, (1b) hardware, and (3) other services. Our main variables of interest are the numbers of new businesses according to the different sectoral definitions.

Data on HEIs come from the German University Statistics of the German Federal Statistical Office, which provides information about every university in Germany ${ }^{3}$ and contains, for example, information on whether a HEI has teaching and research facilities in computer sciences. It also provides information on third-party funding and the general financial budget for computer sciences. This allows us to construct a measure for the quality of computer science facilities by comparing the amount of third-party funding to their actual size as captured by the total budget. HEIs are divided into two categories: regular universities and universities of applied sciences. For both types of universities, we count their number, which we in clude in the analysis as categorical variables. Among the regions hosting a higher education institute with a computer science department the maximum number of regular universities and of universities of applied sciences is two (Table 2). As a control for the size of HEI's computer science departments we also consider the general budget for regular universities and universities of applied sciences in the respective region. ${ }^{4}$

Data on regional specializations were obtained from the Establishment History Panel which is based on German employment statistics. This dataset contains every German establishment that employs at least one person that is obliged to pay social insurance contributions [Spengler, 2008]. With this dataset, it is possible to identify regional specializations in IT-related manufacturing and services that we consider in our analysis. More precisely, we used the total number of employees in both IT-related industries and the share of IT-related services within this overall employment as a percentage.

In the empirical assessment we investigate the time period of 2009-2016. Since we are interested in the recent developments of start-up activity, we take the average values for the regional determinants for the period of 2000-2008 to explain the average start-up

\footnotetext{
${ }^{3}$ https://www.destatis.de/DE/Themen/Gesellschaft-Umwelt/Bildung-Forschung-Kultur/Hochschulen/_inhalt.html. For details, see also [Fritsch, Aamoucke, 2013].

${ }^{4}$ We have no information on the number of students and professors in computer science, which would have been a good alternative indicator.
} 


\section{Figure 1. IT Start-up Rates per 10,000 Working} Adults in Germany between 2009 and 2016

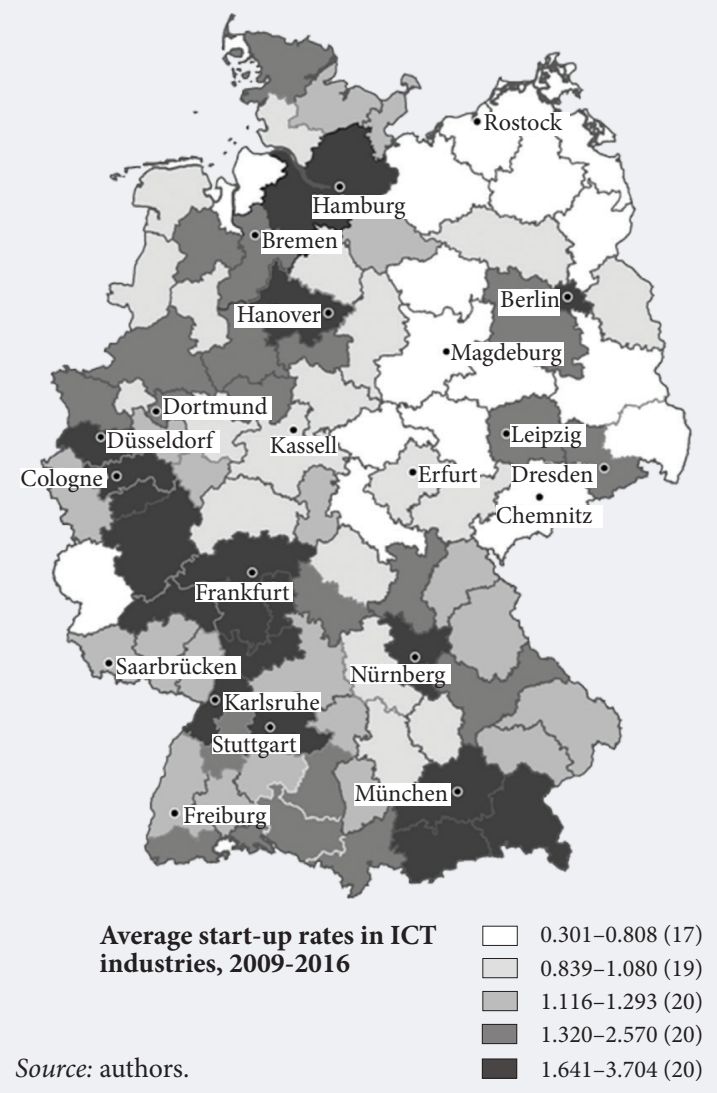

rate in the different IT sectors in the period 20092016. By averaging over these periods, our results are not driven by the impact of the economic crisis in 2008 and we avoid a simultaneity bias.

We use planning regions to create the spatial framework for our empirical analyses. There are 97 German planning regions that represent functionally integrated units comparable to labor-market areas in the United States. The functional economic region of the cities of Hamburg and Bremen comprises also the adjacent planning regions which are merged accordingly. ${ }^{5}$ By doing so, we are left with a total of 93 planning regions in Germany, 71 in the area formerly known as West Germany, and 22 in the former East.

\section{The Regional Distribution of IT Start-ups in Germany}

Most German IT start-ups are in IT software, other IT services, and IT retail \& leasing (see Table 2). Only about 3.7 percent are producing IT hardware. There is great variation in the average yearly number of IT start-ups across regions. Not surprisingly, the number of start-ups in IT industries is much larger in big cities than in rural areas. A simple reason for this phenomenon is that there are more potential founders to be found within the larger workforce of cities. In order to compare the levels of new business formation across regions we calculated start-up rates. The regional start-up rate in IT industries is the average

Table 2. Summary Statistics

\begin{tabular}{|c|c|c|c|c|}
\hline & Mean & $\begin{array}{l}\text { Standard } \\
\text { deviation }\end{array}$ & Minimum & Maximum \\
\hline \multicolumn{5}{|c|}{ Number of IT start-ups } \\
\hline All & 88.323 & 129.867 & 4 & 776 \\
\hline Software, hardware \& consulting & 68.763 & 108.723 & 2 & 671 \\
\hline Hardware & 3.366 & 4.045 & 0 & 23 \\
\hline Software & 32.419 & 54.069 & 1 & 368 \\
\hline Other services & 33.118 & 51.557 & 1 & 280 \\
\hline Retail \& leasing & 19.602 & 23.019 & 2 & 109 \\
\hline \multicolumn{5}{|c|}{ Number of universities with computer science education \& research } \\
\hline Regular universities & 0.677 & 0.628 & 0 & 2 \\
\hline Universities of applied sciences & 0.699 & 0.586 & 0 & 2 \\
\hline \multicolumn{5}{|c|}{ Size of computer science department } \\
\hline Regular universities & 6985.305 & 6009.895 & 12.718 & 29415.537 \\
\hline Universities of applied sciences & 1875.802 & 1455.731 & 11.657 & 5575.184 \\
\hline \multicolumn{5}{|c|}{ Third-party funding intensity of computer science education \& research } \\
\hline Regular universities & 1708.215 & 2059.328 & 0 & 12725.612 \\
\hline Universities of applied sciences & 452.137 & 1487.645 & 0 & 13464.313 \\
\hline \multicolumn{5}{|c|}{ Other indicators } \\
\hline Employment share manufacturing IT hardware & 4395.527 & 7197.911 & 145.222 & 44668.332 \\
\hline Employment share IT services & 0.887 & 0.147 & 0.276 & 0.997 \\
\hline Population density & 5.33 & 0.754 & 3.887 & 8.246 \\
\hline
\end{tabular}

Hamburg is merged with the region of Schleswig-Holstein South and Hamburg-Umland South and Bremen is merged with Bremen-Umland. Berlin could be also merged with adjacent planning regions which implies that the merged area is much larger than the functional economic area of Berlin. 
Table 3. Determinants of IT Start-up Activity in 2009-2016

\begin{tabular}{|c|c|c|c|c|c|c|}
\hline & \multirow{3}{*}{$\begin{array}{l}\text { I } \\
\text { All IT start- } \\
\text { ups }\end{array}$} & II & III & IV & $\mathrm{V}$ & \multirow{3}{*}{$\begin{array}{c}\mathrm{VI} \\
\text { IT retail \& leasing }\end{array}$} \\
\hline & & \multicolumn{4}{|c|}{ IT Software, Hardware, \& Consulting } & \\
\hline & & All & IT hardware & IT software & $\begin{array}{l}\text { Other IT } \\
\text { services }\end{array}$ & \\
\hline \multicolumn{7}{|c|}{ Number of universities of applied science with computer science education \& research } \\
\hline No university $($ Yes $=0)$ & Ref & Ref & Ref & Ref & Ref & Ref \\
\hline One university (Yes=1) & $\begin{array}{l}0.619^{* * *} \\
(0.182)\end{array}$ & $\begin{array}{l}0.451^{\star *} \\
(0.214)\end{array}$ & $\begin{array}{c}0.249 \\
(0.344)\end{array}$ & $\begin{array}{l}0.617^{\star * *} \\
(0.224)\end{array}$ & $\begin{array}{c}0.313 \\
(0.278)\end{array}$ & $\begin{array}{l}1.024^{\star * *} \\
(0.243)\end{array}$ \\
\hline Two universities (Yes=1) & $\begin{array}{c}0.913^{* * *} \\
(0.247)\end{array}$ & $\begin{array}{c}0.779^{* * *} \\
(0.279)\end{array}$ & $\begin{array}{c}0.625 \\
(0.417)\end{array}$ & $\begin{array}{c}0.825^{* * *} \\
(0.298)\end{array}$ & $\begin{array}{l}0.747^{\star *} \\
(0.347)\end{array}$ & $\begin{array}{l}1.200^{* * *} \\
(0.316)\end{array}$ \\
\hline \multicolumn{7}{|c|}{ Number of regular universities with computer science education \& research } \\
\hline No university (Yes=0) & Ref & Ref & Ref & Ref & Ref & Ref \\
\hline One university (Yes=1) & $\begin{array}{l}0.694^{\star *} \\
(0.277)\end{array}$ & $\begin{array}{c}0.467 \\
(0.318)\end{array}$ & $\begin{array}{l}0.614^{\star} \\
(0.365)\end{array}$ & $\begin{array}{c}0.521 \\
(0.356)\end{array}$ & $\begin{array}{c}0.405 \\
(0.313)\end{array}$ & $\begin{array}{l}1.410^{* * *} \\
(0.268)\end{array}$ \\
\hline Two universities (Yes=1) & $\begin{array}{l}0.855^{\star * *} \\
(0.295)\end{array}$ & $\begin{array}{l}0.613^{\star} \\
(0.330)\end{array}$ & $\begin{array}{l}0.803^{\star *} \\
(0.393)\end{array}$ & $\begin{array}{l}0.689^{*} \\
(0.368)\end{array}$ & $\begin{array}{c}0.507 \\
(0.333)\end{array}$ & $\begin{array}{l}1.566^{\star * *} \\
(0.319)\end{array}$ \\
\hline \multicolumn{7}{|c|}{ Size of computer science department at universities } \\
\hline Regular universities & $\begin{array}{l}-0.0298 \\
(0.0498)\end{array}$ & $\begin{array}{l}-0.00689 \\
(0.0537)\end{array}$ & $\begin{array}{c}0.0318 \\
(0.0730)\end{array}$ & $\begin{array}{l}0.00433 \\
(0.0626) \\
\end{array}$ & $\begin{array}{l}-0.00986 \\
(0.0516)\end{array}$ & $\begin{array}{l}-0.126^{* *} \\
(0.0516)\end{array}$ \\
\hline Universities of applied sciences & $\begin{array}{c}-0.0896^{* * *} \\
(0.0324)\end{array}$ & $\begin{array}{l}-0.0561 \\
(0.0375)\end{array}$ & $\begin{array}{l}-0.0379 \\
(0.0496)\end{array}$ & $\begin{array}{l}-0.0764^{*} \\
(0.0397)\end{array}$ & $\begin{array}{l}-0.0444 \\
(0.0479)\end{array}$ & $\begin{array}{c}-0.177^{\star * *} \\
(0.0371)\end{array}$ \\
\hline \multicolumn{7}{|c|}{ Third-party funding intensity of computer science education \& research at universities } \\
\hline Regular universities & $\begin{array}{l}-0.0682 \\
(0.0512) \\
\end{array}$ & $\begin{array}{l}-0.0655 \\
(0.0547) \\
\end{array}$ & $\begin{array}{l}-0.126^{*} \\
(0.0673) \\
\end{array}$ & $\begin{array}{l}-0.0714 \\
(0.0563) \\
\end{array}$ & $\begin{array}{l}-0.0677 \\
(0.0554)\end{array}$ & $\begin{array}{l}-0.0544 \\
(0.0503) \\
\end{array}$ \\
\hline Universities of applied sciences & $\begin{array}{c}-0.00618 \\
(0.0204)\end{array}$ & $\begin{array}{l}-0.0199 \\
(0.0240)\end{array}$ & $\begin{array}{l}-0.0152 \\
(0.0272)\end{array}$ & $\begin{array}{l}-0.0216 \\
(0.0272)\end{array}$ & $\begin{array}{l}-0.0176 \\
(0.0263)\end{array}$ & $\begin{array}{c}0.0314 \\
(0.0208)\end{array}$ \\
\hline \multicolumn{7}{|c|}{ Other indicators } \\
\hline $\begin{array}{l}\text { Number of employees in IT } \\
\text { manufacturing \& services }\end{array}$ & $\begin{array}{l}0.603^{* * *} \\
(0.0608)\end{array}$ & $\begin{array}{l}0.623^{* * *} \\
(0.0661) \\
\end{array}$ & $\begin{array}{l}0.477^{\star * *} \\
(0.0770)\end{array}$ & $\begin{array}{l}0.625^{\star * *} \\
(0.0754)\end{array}$ & $\begin{array}{l}0.650^{* * *} \\
(0.0722) \\
\end{array}$ & $\begin{array}{l}0.571^{\star * *} \\
(0.0709)\end{array}$ \\
\hline $\begin{array}{l}\text { Employment IT service/Employment IT } \\
\text { manufacturing \& services }\end{array}$ & $\begin{array}{c}0.552^{* * *} \\
(0.121)\end{array}$ & $\begin{array}{l}0.595^{\star * *} \\
(0.141)\end{array}$ & $\begin{array}{l}0.320 \\
(0.218)\end{array}$ & $\begin{array}{c}0.577^{* * *} \\
(0.150)\end{array}$ & $\begin{array}{c}0.677^{* * *} \\
(0.158)\end{array}$ & $\begin{array}{l}0.451^{\star * *} \\
(0.138)\end{array}$ \\
\hline Population density & $\begin{array}{l}0.245^{\star * *} \\
(0.0885)\end{array}$ & $\begin{array}{l}0.231^{\star *} \\
(0.107)\end{array}$ & $\begin{array}{l}0.255^{\star *} \\
(0.102)\end{array}$ & $\begin{array}{l}0.245^{\star *} \\
(0.123)\end{array}$ & $\begin{array}{l}0.226^{\star *} \\
(0.0992)\end{array}$ & $\begin{array}{l}0.274^{* * *} \\
(0.0650)\end{array}$ \\
\hline Constant & $\begin{array}{c}-1.735^{* * *} \\
(0.305) \\
\end{array}$ & $\begin{array}{l}-2.083^{* * *} \\
(0.361) \\
\end{array}$ & $\begin{array}{l}-3.960^{* * *} \\
(0.375) \\
\end{array}$ & $\begin{array}{c}-3.016^{* * *} \\
(0.417) \\
\end{array}$ & $\begin{array}{l}-2.900^{* * *} \\
(0.350) \\
\end{array}$ & $\begin{array}{l}-3.053^{* * *} \\
(0.302) \\
\end{array}$ \\
\hline Number of observations & 93 & 93 & 93 & 93 & 93 & 93 \\
\hline Log likelihood & -388.2 & -370 & -151.9 & -309.7 & -309.2 & -275.2 \\
\hline Pseudo R-squared & 0.239 & 0.240 & 0.290 & 0.259 & 0.264 & 0.256 \\
\hline \multicolumn{7}{|c|}{$\begin{array}{l}\text { Notes: Negative binomial regressions. The dependent variable is the number of start-ups in the respective industry. Robust standard errors in parentheses. } \\
\star * * \text { : statistically significant at the } 1 \% \text { level; }{ }^{*}: \text { statistically significant at the } 5 \% \text { level; }{ }^{*} \text { statistically significant at the } 10 \% \text { level. } \\
\text { Source: authors. }\end{array}$} \\
\hline
\end{tabular}

yearly number of new businesses that emerged in the 2009-2016 period divided by the number of people in the regional workforce (in thousands). The regional workforce is the population aged between 18 and 64 years old.

Figure 1 shows that, even when controlling for the size of regional workforce, start-up rates in IT industries tend to be considerably higher in regions with larger cities such as Berlin, Frankfurt, Hamburg, Munich, and Stuttgart than in less densely populated areas. There is a corridor of regions with high levels of new business formation in IT industries along the Rhine from Duesseldorf to Karlsruhe. It is quite notable that IT start-up rates tend to be particularly low in East Germany, the formerly socialist GDR. This observation corresponds to a generally low level of new business formation in this part of the country in the period of analysis.

There may be a number of reasons for higher IT start-up rates in large cities. First, start-ups in IT industries require knowledge that may be regionally bounded, particularly if this knowledge is tacit in nature [Boschma, 2005]. Since all larger cities in Germany host at least one HEI that often has special departments for computer science, this knowledge is more likely to be present in these regions than in rural ones. Second, research in computer science may be an important source of entrepreneurial opportunities [Acs et al., 2009; 2013]. As previously mentioned, the founders of new businesses show a pronounced tendency to set up their venture close to their residence. Accordingly, new businesses tend to be locat- 


\begin{tabular}{|c|c|c|c|c|c|c|c|c|c|c|c|c|c|c|c|c|}
\hline$\stackrel{\sqrt[n]{\Xi}}{ }$ & & & & & & & & & & & & & & & - & \\
\hline$\Xi$ & & & & & & & & & & & & & & - & 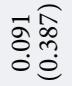 & \\
\hline $\bar{\Xi}$ & & & & & & & & & & & & & - & 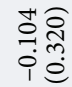 & 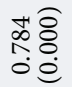 & \\
\hline$\Xi$ & & & & & & & & & & & & - & 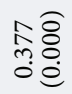 & 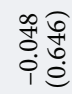 & 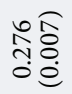 & \\
\hline$\Xi$ & & & & & & & & & & & - & 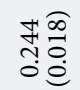 & 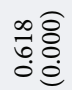 & 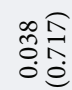 & 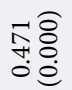 & \\
\hline$\stackrel{\Xi}{\Xi}$ & & & & & & & & & & - & 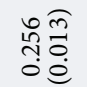 & 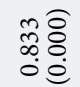 & 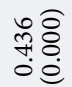 & 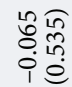 & 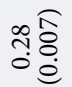 & \\
\hline $\bar{\sigma}$ & & & & & & & & & - & 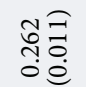 & 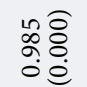 & 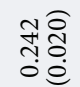 & 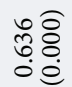 & 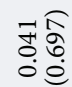 & 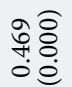 & \\
\hline$\underline{\infty}$ & & & & & & & & - & 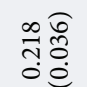 & $\begin{array}{l}\bar{\delta} \widehat{\circ} \\
\text { o. }\end{array}$ & 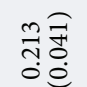 & 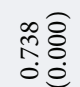 & 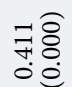 & 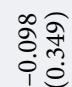 & 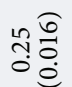 & \\
\hline$\Sigma$ & & & & & & & - & 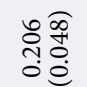 & 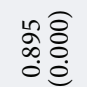 & 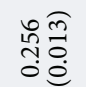 & 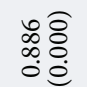 & 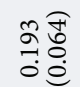 & 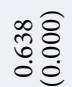 & 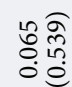 & 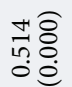 & \\
\hline$\sqrt{6}$ & & & & & & $\tau$ & 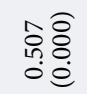 & 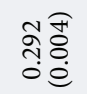 & 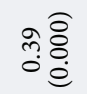 & 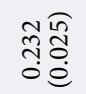 & 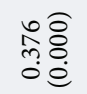 & 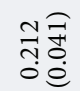 & $\begin{array}{l}\infty \\
\text { to } \\
\vdots \\
0\end{array}$ & 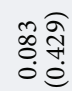 & 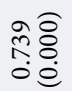 & \\
\hline$\sqrt{6}$ & & & & & - & 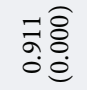 & 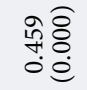 & 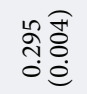 & 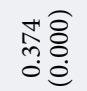 & 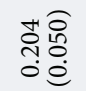 & 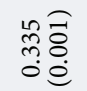 & 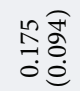 & $\begin{array}{l}\text { to. } \\
\text { o. }\end{array}$ & $\begin{array}{l}\text { no } \\
\hat{0} \\
0 \\
0\end{array}$ & 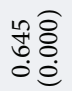 & \\
\hline$\Xi$ & & & & - & 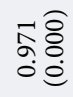 & $\begin{array}{l}\infty \\
\infty \\
\infty \\
0 \\
0\end{array}$ & 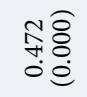 & 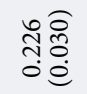 & 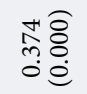 & 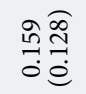 & $\begin{array}{c}\text { लై } \\
\text { o. }\end{array}$ & 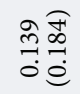 & $\begin{array}{l}\hat{h} \\
\hat{b} \\
\dot{0}\end{array}$ & 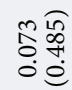 & 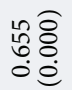 & \\
\hline $\bar{m}$ & & & - & $\begin{array}{l}\text { ț } \\
\text { o. } \\
\circ \\
\end{array}$ & 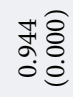 & \begin{tabular}{l}
$\infty$ \\
$\infty$ \\
$\infty$ \\
$\infty$ \\
\hdashline
\end{tabular} & 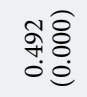 & 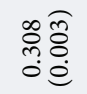 & 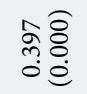 & 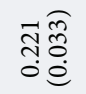 & 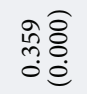 & $\begin{array}{l}\infty \\
\infty \\
0.0 \\
0 .\end{array}$ & 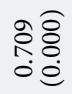 & 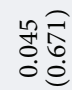 & $\begin{array}{l}\vec{b} 0 \\
\text { ○. }\end{array}$ & \\
\hline $\bar{\Xi}$ & & $\tau$ & 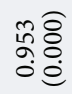 & 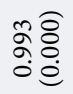 & $\begin{array}{l}\text { moे } \\
\text { o. }\end{array}$ & 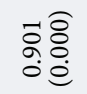 & $\begin{array}{l}\text { 동 } \\
\text { fo }\end{array}$ & 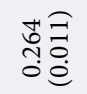 & $\begin{array}{l}\infty \widehat{o} \\
0 \\
0 \\
0\end{array}$ & 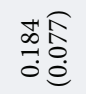 & শ্ & 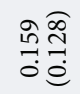 & 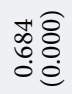 & 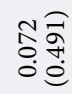 & 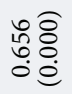 & \\
\hline$\Xi$ & - & 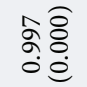 & $\begin{array}{l}\text { బू. } \\
\text { o. }\end{array}$ & 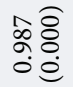 & $\begin{array}{l}\text { Sूठ } \\
\text { o. }\end{array}$ & 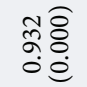 & 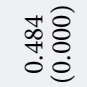 & 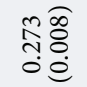 & 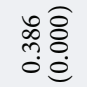 & 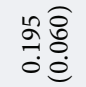 & $\begin{array}{l}\overrightarrow{\tilde{m}} \widehat{\bar{\delta}} \\
0 \\
0\end{array}$ & $\begin{array}{l}\text { 등 } \\
\text { 응 }\end{array}$ & 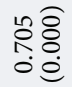 & 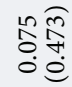 & \begin{tabular}{l} 
कôे \\
: \\
\hdashline
\end{tabular} & \\
\hline & 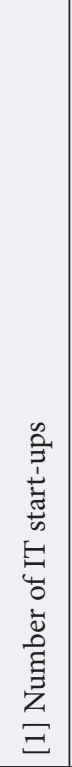 & 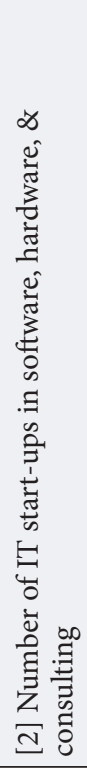 & 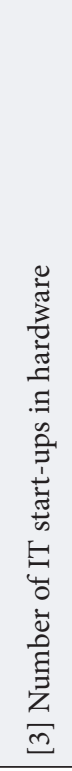 & 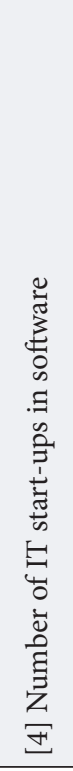 & 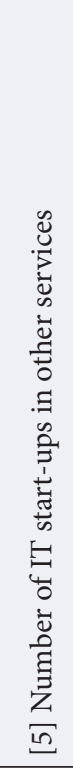 & 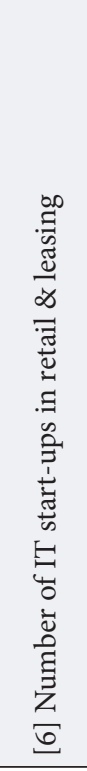 & 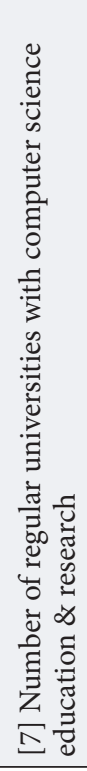 & 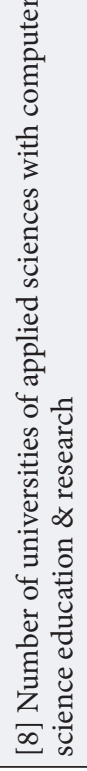 & 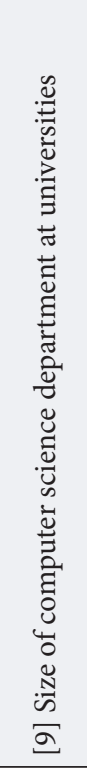 & 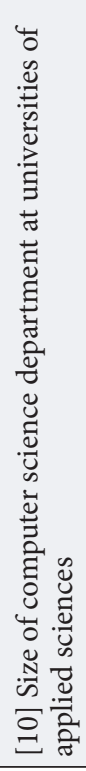 & 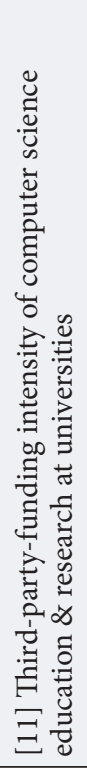 & 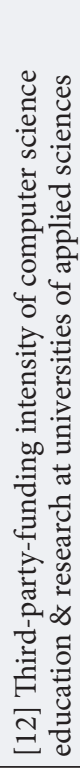 & 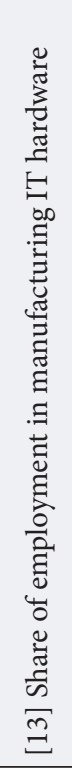 & 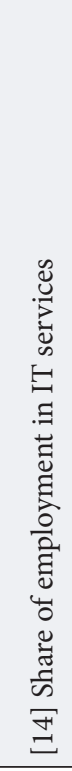 & 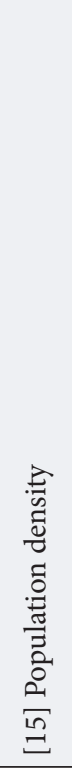 & 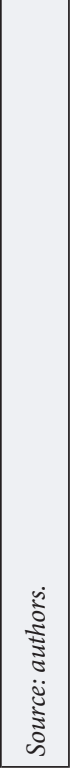 \\
\hline
\end{tabular}




\section{Table 5. Robustness Check. Determinants of IT Start-up Activity: University Effects Only}

\begin{tabular}{|c|c|c|c|c|c|c|}
\hline & \multirow{3}{*}{$\begin{array}{l}\text { I } \\
\text { All IT start- } \\
\text { ups }\end{array}$} & II & III & IV & $\mathrm{V}$ & \multirow{3}{*}{$\begin{array}{c}\text { VI } \\
\text { IT retail \& } \\
\text { leasing }\end{array}$} \\
\hline & & \multicolumn{4}{|c|}{ Startups in IT Software, Hardware, \& Consulting } & \\
\hline & & All & IT hardware & IT software & $\begin{array}{c}\text { Other IT } \\
\text { services }\end{array}$ & \\
\hline \multicolumn{7}{|c|}{ Number of universities of applied science with computer science education \& research } \\
\hline No university $($ Yes $=0)$ & Ref & Ref & Ref & Ref & Ref & Ref \\
\hline One university (Yes=1) & $\begin{array}{c}0.452^{\star * *} \\
(0.165)\end{array}$ & $\begin{array}{c}0.459^{* * *} \\
(0.173)\end{array}$ & $\begin{array}{c}0.245 \\
(0.176) \\
\end{array}$ & $\begin{array}{c}0.492^{* * *} \\
(0.177)\end{array}$ & $\begin{array}{c}0.438^{\star *} \\
(0.183) \\
\end{array}$ & $\begin{array}{c}0.425^{\star * *} \\
(0.158)\end{array}$ \\
\hline Two universities (Yes=1) & $\begin{array}{c}1.170^{\star * *} \\
(0.361)\end{array}$ & $\begin{array}{c}1.216^{\star * *} \\
(0.359)\end{array}$ & $\begin{array}{c}1.014^{* * *} \\
(0.341)\end{array}$ & $\begin{array}{c}1.108^{* * *} \\
(0.363)\end{array}$ & $\begin{array}{c}1.320^{* * *} \\
(0.362)\end{array}$ & $\begin{array}{l}0.989^{* *} \\
(0.386)\end{array}$ \\
\hline \multicolumn{7}{|c|}{ Number of regular universities with computer science education \& research } \\
\hline No university (Yes=0) & Ref & Ref & Ref & Ref & Ref & Ref \\
\hline One university (Yes=1) & $\begin{array}{c}0.788^{* * *} \\
(0.171)\end{array}$ & $\begin{array}{c}0.827^{\star * *} \\
(0.180)\end{array}$ & $\begin{array}{c}0.616^{* * *} \\
(0.148)\end{array}$ & $\begin{array}{c}0.922^{* * *} \\
(0.179)\end{array}$ & $\begin{array}{c}0.762^{\star * *} \\
(0.194)\end{array}$ & $\begin{array}{c}0.661^{* * *} \\
(0.165)\end{array}$ \\
\hline Two universities (Yes $=1$ ) & $\begin{array}{c}2.017^{\star * *} \\
(0.418)\end{array}$ & $\begin{array}{c}2.103^{* * *} \\
(0.453)\end{array}$ & $\begin{array}{c}1.585^{\star * *} \\
(0.339)\end{array}$ & $\begin{array}{c}2.282^{* * *} \\
(0.492)\end{array}$ & $\begin{array}{c}1.972^{* * *} \\
(0.422)\end{array}$ & $\begin{array}{c}1.705^{\star * *} \\
(0.319)\end{array}$ \\
\hline \multicolumn{7}{|c|}{ Other indicators } \\
\hline Constant & $\begin{array}{c}3.306^{* * *} \\
(0.130)\end{array}$ & $\begin{array}{c}2.997^{\star * *} \\
(0.138)\end{array}$ & $\begin{array}{l}0.377^{\star *} \\
(0.152)\end{array}$ & $\begin{array}{c}2.145^{\star * *} \\
(0.145)\end{array}$ & $\begin{array}{c}2.327^{\star * *} \\
(0.147)\end{array}$ & $\begin{array}{c}1.997^{\star * *} \\
(0.127)\end{array}$ \\
\hline Number of observations & 93 & 93 & 93 & 93 & 93 & 93 \\
\hline Log likelihood & -473 & -449.7 & -187.5 & -379.6 & -385.3 & -341.4 \\
\hline Pseudo R-squared & 0.0726 & 0.0768 & 0.124 & 0.0916 & 0.0824 & 0.0774 \\
\hline
\end{tabular}

ed in geographic proximity of the knowledge sources that are highly concentrated in the larger cities. Third, there are larger volumes of demand in large cities that may be particularly relevant for IT services that require face-to-face contact.

\section{Empirical Analysis}

To identify the factors that determine the regional emergence of innovative start-ups, we performed multivariate analyses. Our dependent variable is the average number of innovative start-ups in a region over the 2009-2016 period. We run these models for all IT start-ups as well as for a number of sub-categories: IT software, IT hardware, IT consulting, and IT retail \& leasing. Due to the count character of the independent variable, the number of start-ups, we employed a negative binomial estimation technique. In order to avoid any reverse causality issues, all in dependent variables are yearly averages for the period 2000-2008. While the numbers of regular universities and universities of applied sciences are integer values, all other independent variables are log-transformed. ${ }^{6}$

Table 3 shows the main results. We find significantly positive relationships between the total number of IT start-ups and the number of regular universities as well as universities of applied sciences. According to the estimated coefficients, the effect is more pro- nounced if a region is hosting two universities of applied sciences or two regular universities. For both types of universities, having two of them is associated with one more IT start-up per 10,000 people in the workforce as compared to regions that do not host a HEI. The effect for hosting at least one university of applied science or a regular university is around 0.5 more start-ups per year as compared to regions without HEIs. In order to test whether the findings for the presence of HEIs are driven by multicollinearity with size measures and other regional variables like population density, we ran models with only the indicators for both types of universities and no other indicator related to size. The results (see Table 4) are rather similar to those of the full model of Table 3.

Somewhat surprisingly the size of universities of applied sciences is negatively related to overall startup activity in the IT sector while neither the size of computer science departments at regular universities nor the third-party funding intensity are significantly related to IT start-up activity. This result clearly suggests that the size of local computer science departments does not play a role.

The regional number of employees in the IT sector has a positive effect on IT start-up activity. This effect is more pronounced if IT employment is concentrated in IT services. A 10 percent increase in this employ-

\footnotetext{
${ }^{6}$ There are no negative values. The original variables are scaled up to the extent that the smallest values that are larger than zero assume a positive value after log-transformation. Values that were zero before this transformation are then kept as zeros. More precisely, we multiplied size and third-party funding intensity of computer science education \& research by the factor of 10,000 .
} 


\section{Table 6. Robustness check. Determinants of IT Start-up Activity: No Control for Population Density}

\begin{tabular}{|c|c|c|c|c|c|c|}
\hline & \multirow{3}{*}{$\begin{array}{l}\text { I } \\
\text { All IT } \\
\text { start-ups }\end{array}$} & II & III & IV & $\mathrm{V}$ & \multirow{3}{*}{$\begin{array}{c}\text { VI } \\
\text { IT retail \& leasing }\end{array}$} \\
\hline & & \multicolumn{4}{|c|}{ Startups in IT Software, Hardware, \& Consulting } & \\
\hline & & All & IT hardware & IT software & $\begin{array}{l}\text { Other IT } \\
\text { services }\end{array}$ & \\
\hline \multicolumn{7}{|c|}{ Number of universities of applied science with computer science education \& research } \\
\hline No university $($ Yes $=0)$ & Ref & Ref & Ref & Ref & Ref & Ref \\
\hline One university (Yes=1) & $\begin{array}{c}0.649^{* * *} \\
(0.181)\end{array}$ & $\begin{array}{l}0.474^{\star *} \\
(0.207) \\
\end{array}$ & $\begin{array}{c}0.133 \\
(0.327) \\
\end{array}$ & $\begin{array}{c}0.634^{* * *} \\
(0.219)\end{array}$ & $\begin{array}{c}0.324 \\
(0.261) \\
\end{array}$ & $\begin{array}{l}1.044^{\star * *} \\
(0.262)\end{array}$ \\
\hline Two universities (Yes=1) & $\begin{array}{c}0.899^{* * *} \\
(0.229)\end{array}$ & $\begin{array}{c}0.758^{\star * *} \\
(0.253) \\
\end{array}$ & $\begin{array}{c}0.396 \\
(0.391) \\
\end{array}$ & $\begin{array}{c}0.778^{\star * *} \\
(0.263) \\
\end{array}$ & $\begin{array}{l}0.715^{\star *} \\
(0.317)\end{array}$ & $\begin{array}{l}1.152^{\star * *} \\
(0.337)\end{array}$ \\
\hline \multicolumn{7}{|c|}{ Number of regular universities with computer science education \& research } \\
\hline No university (Yes=0) & Ref & Ref & Ref & Ref & Ref & Ref \\
\hline One university (Yes=1) & $\begin{array}{l}0.622^{* *} \\
(0.261) \\
\end{array}$ & $\begin{array}{c}0.391 \\
(0.300) \\
\end{array}$ & $\begin{array}{c}0.536 \\
(0.368) \\
\end{array}$ & $\begin{array}{c}0.446 \\
(0.348) \\
\end{array}$ & $\begin{array}{c}0.333 \\
(0.291) \\
\end{array}$ & $\begin{array}{l}1.351^{* * *} \\
(0.254) \\
\end{array}$ \\
\hline Two universities (Yes=1) & $\begin{array}{c}0.896^{* * *} \\
(0.262)\end{array}$ & $\begin{array}{l}0.649^{\star *} \\
(0.294) \\
\end{array}$ & $\begin{array}{l}0.778^{\star *} \\
(0.385) \\
\end{array}$ & $\begin{array}{l}0.734^{\star *} \\
(0.340) \\
\end{array}$ & $\begin{array}{l}0.536^{*} \\
(0.289) \\
\end{array}$ & $\begin{array}{l}1.612^{\star * *} \\
(0.298)\end{array}$ \\
\hline \multicolumn{7}{|c|}{ Size of computer science department at universities } \\
\hline Regular universities & $\begin{array}{l}-0.0537 \\
(0.0560)\end{array}$ & $\begin{array}{l}-0.0290 \\
(0.0589)\end{array}$ & $\begin{array}{l}7.30 \mathrm{e}-05 \\
(0.0818) \\
\end{array}$ & $\begin{array}{l}-0.0205 \\
(0.0675) \\
\end{array}$ & $\begin{array}{l}-0.0331 \\
(0.0570) \\
\end{array}$ & $\begin{array}{l}-0.156^{* * *} \\
(0.0565)\end{array}$ \\
\hline Universities of applied sciences & $\begin{array}{c}-0.104^{* * *} \\
(0.0338)\end{array}$ & $\begin{array}{l}-0.0691^{\star} \\
(0.0380) \\
\end{array}$ & $\begin{array}{l}-0.0372 \\
(0.0463) \\
\end{array}$ & $\begin{array}{c}-0.0899^{* *} \\
(0.0401)\end{array}$ & $\begin{array}{l}-0.0570 \\
(0.0478) \\
\end{array}$ & $\begin{array}{c}-0.193^{* * *} \\
(0.0405)\end{array}$ \\
\hline \multicolumn{7}{|c|}{ Third-party funding intensity of computer science education \& research at universities } \\
\hline Regular universities & $\begin{array}{l}-0.0373 \\
(0.0500)\end{array}$ & $\begin{array}{l}-0.0356 \\
(0.0520)\end{array}$ & $\begin{array}{l}-0.0887 \\
(0.0680)\end{array}$ & $\begin{array}{l}-0.0398 \\
(0.0530)\end{array}$ & $\begin{array}{l}-0.0373 \\
(0.0543)\end{array}$ & $\begin{array}{l}-0.0215 \\
(0.0520)\end{array}$ \\
\hline Universities of applied sciences & $\begin{array}{l}0.000488 \\
(0.0194)\end{array}$ & $\begin{array}{l}-0.0147 \\
(0.0224)\end{array}$ & $\begin{array}{l}-0.0120 \\
(0.0253)\end{array}$ & $\begin{array}{l}-0.0159 \\
(0.0254)\end{array}$ & $\begin{array}{l}-0.0105 \\
(0.0253)\end{array}$ & $\begin{array}{l}0.0421^{\star} \\
(0.0228)\end{array}$ \\
\hline \multicolumn{7}{|c|}{ Other indicators } \\
\hline $\begin{array}{l}\text { Number of employees in IT } \\
\text { manufacturing \& services }\end{array}$ & $\begin{array}{l}0.730^{\star * *} \\
(0.0409) \\
\end{array}$ & $\begin{array}{l}0.743^{\star * *} \\
(0.0442) \\
\end{array}$ & $\begin{array}{l}0.629^{* * *} \\
(0.0573) \\
\end{array}$ & $\begin{array}{l}0.757^{\star * *} \\
(0.0520) \\
\end{array}$ & $\begin{array}{l}0.768^{\star * *} \\
(0.0474) \\
\end{array}$ & $\begin{array}{l}0.717^{\star * *} \\
(0.0515) \\
\end{array}$ \\
\hline $\begin{array}{l}\text { Employment in IT services/ } \\
\text { Employment in IT manufacturing } \\
\text { \& services }\end{array}$ & $\begin{array}{l}0.682^{* * *} \\
(0.126)\end{array}$ & $\begin{array}{l}0.713^{* * *} \\
(0.144)\end{array}$ & $\begin{array}{l}0.438^{*} \\
(0.236)\end{array}$ & $\begin{array}{l}0.703^{* * *} \\
(0.149)\end{array}$ & $\begin{array}{l}0.787^{* * *} \\
(0.165)\end{array}$ & $\begin{array}{l}0.609^{* * *} \\
(0.145)\end{array}$ \\
\hline Population density & Нет & Нет & Нет & Нет & Нет & Нет \\
\hline Constant & $\begin{array}{l}-1.319^{* * *} \\
(0.271) \\
\end{array}$ & $\begin{array}{c}-1.695^{\star * *} \\
(0.297) \\
\end{array}$ & $\begin{array}{c}-3.630^{* * *} \\
(0.388)\end{array}$ & $\begin{array}{c}-2.633^{* * *} \\
(0.346) \\
\end{array}$ & $\begin{array}{c}-2.522^{* * *} \\
(0.319) \\
\end{array}$ & $\begin{array}{c}-2.614^{\star * *} \\
(0.334) \\
\end{array}$ \\
\hline Number of observations & 93 & 93 & 93 & 93 & 93 & 93 \\
\hline Log likelihood & -394.1 & -374.5 & -154.7 & -313.7 & -313.1 & -280.7 \\
\hline Pseudo R-squared & 0.227 & 0.231 & 0.278 & 0.249 & 0.254 & 0.242 \\
\hline
\end{tabular}

ment share is associated with an about 6 percent higher number of overall IT start-ups. Thus, consistent with the knowledge spillover theory of entrepreneurship [Acs et al., 2009; 2013] a high share of employees with industry experience in IT increases the level of new firm formation in that sector. Finally, population density is significantly and positively related to the number of start-ups. A 10 percent higher population density is associated with an about 2.5 percent more IT start-ups. The coefficient estimates for the other variables are relatively similar when population density is excluded from the model (Table 6).

Looking into the IT sub-categories reveals further interesting findings. The effect of HEIs on IT startup activity seems to be driven by IT software and IT retail \& leasing whereas the effect is particularly pronounced for the latter type of IT industries. For
IT hardware, there is only a statistically significant effect for regions having two regular universities. For other IT services, the presence of two universities of applied sciences is significant only. In the models that consider only indicators for the presence of HEIs, all indicators are statistically significant (Table 5).

The size and quality of computer science departments do apparently not matter while the number of employees in the IT sector is positively related to the level of start-up activity in all IT sub-categories. The same applies to specialization in IT services. There is one exception namely that the coefficient estimate for the regional employment share in IT services on the number of start-ups in IT hardware is insignificant. This may be an indication that industry experience in IT software is less relevant for the production of IT hardware. Finally, there is a significantly positive 


\section{Table 7. Robustness Check. Determinants of IT Start-up Activity (log-transformed number of start-ups, OLS)}

\begin{tabular}{|c|c|c|c|c|c|c|}
\hline & \multirow{3}{*}{$\begin{array}{l}\text { I } \\
\text { All IT start- } \\
\text { ups }\end{array}$} & II & III & IV & $\mathrm{V}$ & \multirow{3}{*}{$\begin{array}{l}\mathrm{VI} \\
\text { IT retail \& } \\
\text { leasing }\end{array}$} \\
\hline & & \multicolumn{4}{|c|}{ IT Software, Hardware, \& Consulting } & \\
\hline & & All & IT hardware & IT software & $\begin{array}{c}\text { Other IT } \\
\text { services }\end{array}$ & \\
\hline \multicolumn{7}{|c|}{ Number of universities of applied science with computer science education \& research } \\
\hline No university $($ Yes $=0)$ & Ref & Ref & Ref & Ref & Ref & Ref \\
\hline One university (Yes=1) & $\begin{array}{c}0.748^{* * *} \\
(0.180)\end{array}$ & $\begin{array}{c}0.573^{\star * *} \\
(0.213)\end{array}$ & $\begin{array}{c}0.369 \\
(0.325) \\
\end{array}$ & $\begin{array}{c}0.762^{\text {***}} \\
(0.232)\end{array}$ & $\begin{array}{l}0.467^{\star} \\
(0.245)\end{array}$ & $\begin{array}{c}1.124^{* * *} \\
(0.288)\end{array}$ \\
\hline Two universities $(\mathrm{N}=2$, Yes=1) & $\begin{array}{c}1.089^{\star * *} \\
(0.232)\end{array}$ & $\begin{array}{c}0.951^{\star * *} \\
(0.264)\end{array}$ & $\begin{array}{c}0.729^{*} \\
(0.387) \\
\end{array}$ & $\begin{array}{c}1.010^{* * *} \\
(0.287)\end{array}$ & $\begin{array}{c}0.972^{\star * *} \\
(0.295)\end{array}$ & $\begin{array}{c}1.308^{* * *} \\
(0.340)\end{array}$ \\
\hline \multicolumn{7}{|c|}{ Number of regular universities with computer science education \& research } \\
\hline No university $($ Yes $=0)$ & Ref & Ref & Ref & Ref & Ref & Ref \\
\hline One university (Yes=1) & $\begin{array}{c}0.749^{* * *} \\
(0.236)\end{array}$ & $\begin{array}{l}0.547^{\star} \\
(0.277) \\
\end{array}$ & $\begin{array}{l}0.928^{\star *} \\
(0.450)\end{array}$ & $\begin{array}{l}0.606^{*} \\
(0.310) \\
\end{array}$ & $\begin{array}{l}0.482^{*} \\
(0.256) \\
\end{array}$ & $\begin{array}{c}1.400^{* * *} \\
(0.219)\end{array}$ \\
\hline Two universities $(\mathrm{N}=2$, Yes $=1)$ & $\begin{array}{c}0.901^{* * *} \\
(0.261)\end{array}$ & $\begin{array}{l}0.668^{\star *} \\
(0.289)\end{array}$ & $\begin{array}{l}1.189^{* *} \\
(0.480)\end{array}$ & $\begin{array}{l}0.743^{\star *} \\
(0.320)\end{array}$ & $\begin{array}{l}0.579^{\star *} \\
(0.274)\end{array}$ & $\begin{array}{c}1.634^{* * *} \\
(0.290)\end{array}$ \\
\hline \multicolumn{7}{|c|}{ Size of computer science department at universities } \\
\hline Regular universities & $\begin{array}{l}-0.0489 \\
(0.0527) \\
\end{array}$ & $\begin{array}{l}-0.0292 \\
(0.0553)\end{array}$ & $\begin{array}{l}-0.0504 \\
(0.0919) \\
\end{array}$ & $\begin{array}{l}-0.0246 \\
(0.0615)\end{array}$ & $\begin{array}{l}-0.0362 \\
(0.0546)\end{array}$ & $\begin{array}{l}-0.143^{* *} \\
(0.0603)\end{array}$ \\
\hline Universities of applied sciences & $\begin{array}{c}-0.107^{* * *} \\
(0.0308)\end{array}$ & $\begin{array}{c}-0.0710^{* *} \\
(0.0352)\end{array}$ & $\begin{array}{l}-0.0332 \\
(0.0507)\end{array}$ & $\begin{array}{c}-0.0883^{\star *} \\
(0.0376)\end{array}$ & $\begin{array}{l}-0.0645 \\
(0.0414)\end{array}$ & $\begin{array}{c}-0.190^{* * *} \\
(0.0428)\end{array}$ \\
\hline \multicolumn{7}{|c|}{ Third-party funding intensity of computer science education \& research at universities } \\
\hline $\begin{array}{l}\text { Third-party funding intensity of computer } \\
\text { science education \& research at universities }\end{array}$ & $\begin{array}{l}-0.0487 \\
(0.0496)\end{array}$ & $\begin{array}{l}-0.0446 \\
(0.0521)\end{array}$ & $\begin{array}{l}-0.0683 \\
(0.0852) \\
\end{array}$ & $\begin{array}{l}-0.0449 \\
(0.0542) \\
\end{array}$ & $\begin{array}{l}-0.0385 \\
(0.0535)\end{array}$ & $\begin{array}{l}-0.0332 \\
(0.0532) \\
\end{array}$ \\
\hline $\begin{array}{l}\text { Third-party funding intensity of computer } \\
\text { science education \& research at universities of } \\
\text { applied sciences }\end{array}$ & $\begin{array}{l}-0.00323 \\
(0.0199)\end{array}$ & $\begin{array}{l}-0.0175 \\
(0.0221)\end{array}$ & $\begin{array}{l}-0.00962 \\
(0.0254)\end{array}$ & $\begin{array}{l}-0.0221 \\
(0.0245)\end{array}$ & $\begin{array}{l}-0.0144 \\
(0.0236)\end{array}$ & $\begin{array}{c}0.0342 \\
(0.0226)\end{array}$ \\
\hline \multicolumn{7}{|c|}{ Other indicators } \\
\hline $\begin{array}{l}\text { Number of employees in IT manufacturing \& } \\
\text { services }\end{array}$ & $\begin{array}{l}0.567^{* * *} \\
(0.0591)\end{array}$ & $\begin{array}{l}0.588^{\star * *} \\
(0.0630)\end{array}$ & $\begin{array}{l}0.543^{* * *} \\
(0.0777)\end{array}$ & $\begin{array}{l}0.593^{\star * *} \\
(0.0677)\end{array}$ & $\begin{array}{l}0.590^{* * *} \\
(0.0670)\end{array}$ & $\begin{array}{l}0.529 * * * \\
(0.0690)\end{array}$ \\
\hline $\begin{array}{l}\text { Employment in IT services/Employment in IT } \\
\text { manufacturing \& services }\end{array}$ & $\begin{array}{l}0.510^{* * *} \\
(0.124)\end{array}$ & $\begin{array}{c}0.556^{* * *} \\
(0.137) \\
\end{array}$ & $\begin{array}{c}0.306 \\
(0.260) \\
\end{array}$ & $\begin{array}{l}0.524^{\star * *} \\
(0.135)\end{array}$ & $\begin{array}{l}0.612^{* * *} \\
(0.154)\end{array}$ & $\begin{array}{c}0.380^{* * *} \\
(0.142)\end{array}$ \\
\hline Population density & $\begin{array}{l}0.288^{* * *} \\
(0.0896)\end{array}$ & $\begin{array}{l}0.275^{\star *} \\
(0.108)\end{array}$ & $\begin{array}{c}0.105 \\
(0.131) \\
\end{array}$ & $\begin{array}{l}0.272^{\star *} \\
(0.124)\end{array}$ & $\begin{array}{l}0.298^{\star * *} \\
(0.0981) \\
\end{array}$ & $\begin{array}{l}0.306^{* * *} \\
(0.0704)\end{array}$ \\
\hline Constant & $\begin{array}{c}-1.783^{\star * *} \\
(0.337) \\
\end{array}$ & $\begin{array}{c}-2.160^{\star * *} \\
(0.395) \\
\end{array}$ & $\begin{array}{c}-3.955^{\star * *} \\
(0.452) \\
\end{array}$ & $\begin{array}{c}-3.056^{* * *} \\
(0.447) \\
\end{array}$ & $\begin{array}{c}-2.959^{* * *} \\
(0.383) \\
\end{array}$ & $\begin{array}{c}-3.006^{\star * *} \\
(0.308)\end{array}$ \\
\hline Number of observations & 93 & 93 & 93 & 93 & 93 & 93 \\
\hline R-squared & 0.901 & 0.890 & 0.741 & 0.872 & 0.880 & 0.844 \\
\hline
\end{tabular}

relationship between population density and start-up activity in the different IT sub-categories. The coefficient estimates do not change remarkably when population density is removed from the models (see Table 6).

As a final robustness check, we replicated the results of our baseline models of Table 2 using OLS regression with the log-transformed number of start-ups as an outcome variable. The results are in line with our baseline models. One notable exception is that the effects for HEIs come out more clearly. Furthermore, there is no positive effect for population density in the models on start-up activity in IT hardware (Table 7). Altogether, based on the coefficient estimates and the results of the robustness checks we conclude that regional presence of HEIs plays a more important role than population density. HEIs seem to exert an effect on start-up activity that is independent of population density and the associated agglomeration economies.

\section{Summary and Conclusions}

The main results of our empirical analysis of the determinants of regional new business formation in the IT sector can be summarized as follows.

- First, IT start-ups are concentrated in large cities. The propensity of an IT start-up to emerge in a rural area is rather low.

- Second, a main reason for the geographic concentration of new IT businesses is the presence of HEIs with education and research in computer science. HEIs with computer science departments may be important for the qualification of the regional workforce in IT and as a source of 
new knowledge that opens up promising entrepreneurial opportunities in this technological field.

- Third, it is not the size of the computer science departments at HEIs nor the quality of the research but more their sheer presence that is important for the number of regional IT start-ups.

- Fourth, there is a significantly positive relationship between the regional employment share in IT services and the number of IT start-ups. This is a further indication of the important role industry-specific knowledge plays in the level of entrepreneurship in a certain sector.

- Fifth, population density and resulting agglomeration economies may be conducive to the regional formation of new IT businesses. The effect of population density as such is, however, much smaller than for the presence of HEIs.

All in all, these results are consistent with the knowledge spillover theory of entrepreneurship [Acs et al.,
2009; 2013] that emphasizes the role of knowledge for new business formation. Accordingly, strengthening the regional knowledge base should be a key strategy of any policy that aims at stimulating the number of regional IT start-ups. Since there are good reasons why HEIs in computer sciences are located in larger cities, such high-density regions have a locational advantage in this sector as compared to rural regions.

A limitation of the study is the fact that the data for start-ups do not necessarily cover very small firms such as new businesses that consist of only the founder and have no further employees (solo entrepreneurship). This neglect of micro firms can, however, be also regarded an advantage in the sense that the analysis includes start-ups that may have an effect on the development of the respective region. Future research should complement our findings with a qualitative assessment of location choices of IT founders and the role that local conditions such as the regional knowledge base have for their decision-making and the venture process.

\section{References}

Acs Z.J., Braunerhjelm P., Audretsch D.B., Carlsson B. (2009) The Knowledge Spillover Theory of Entrepreneurship. Small Business Economics, vol. 32, pp. 15-30. Available at: https://doi.org/10.1007/s11187-008-9157-3, accessed 21.04.2019.

Acs Z.J., Audretsch D.B., Lehmann E. (2013) The knowledge spillover theory of entrepreneurship. Small Business Economics, vol. 41, pp. 767-774. Available at: https://doi.org/10.1007/s11187-013-9505-9, accessed 21.04.2019.

Asheim B.T., Gertler M.S. (2006) The geography of innovation: Regional innovation systems. The Oxford Handbook of Innovation (eds. J. Fagerberg, D.C. Mowery, R.R. Nelson), Oxford: Oxford University Press, pp. 291-317. Available at: https://doi.org/10.1093/ oxfordhb/9780199286805.003.0011, accessed 21.04.2019.

Bersch J., Gottschalk S., Müller B., Niefert M. (2014) The Mannheim Enterprise Panel (MUP) and Firm Statistics for Germany (ZEW Discussion Paper 14-104), Mannheim: ZEW. Available at: http://ftp.zew.de/pub/zew-docs/dp/dp14104.pdf, accessed 21.04.2019.

Boschma R. (2005) Proximity and innovation: A critical assessment. Regional Studies, vol. 39, pp. 61-74. Available at: https://doi.org/10.10 80/00343404.2016.1254767, accessed 21.04.2019.

Dahl M.S., Sorenson O. (2009) The Embedded Entrepreneur. European Management Review, vol. 6, pp. 172-181. Available at: https://doi. org/10.1057/emr.2009.14, accessed 21.04.2019.

Figueiredo O., Guimaraes P., Woodward D. (2002) Home-Field Advantage: Location Decisions of Portuguese Entrepreneurs. Journal of Urban Economics, vol. 52, pp. 341-361. Available at: https://doi.org/10.1016/S0094-1190(02)00006-2, accessed 21.04.2019.

Fritsch M., Aamoucke R. (2013) Regional Public Research, Higher Education, and Innovative Start-ups: An Empirical Investigation. Small Business Economics, vol. 41, pp. 865-885. Available at: https://doi.org/10.1007/s11187-013-9510-z, accessed 21.04.2019.

Fritsch M., Aamoucke R. (2017) Fields of Knowledge in Higher Education Institutions, and Innovative Start-Ups - An Empirical Investigation. Papers in Regional Science, vol. 96, pp. S1-S27. Available at: https://doi.org/10.1111/pirs.12175, accessed 21.04.2019.

Glaeser E., Sacerdote B. (2000) The Social Consequences of Housing. Journal of Housing Economics, vol. IX, pp. 1-23.

Helsley R.W., Strange W.C. (2011) Entrepreneurs and Cities: Complexity, Thickness and Balance. Regional Science and Urban Economics, vol. 41, pp. 550-559. Available at: https://doi.org/10.1016/j.regsciurbeco.2011.04.001, accessed 21.04.2019.

Jacobs J. (1969) The Economy of Cities, New York (NY): Random House.

Spengler A. (2008) The Establishment History Panel. Schmollers Jahrbuch / Journal of Applied Social Science Studies, vol. 128, pp. 501-509. Available at: https://doi.org/10.3790/schm.128.3.501, accessed 21.04.2019.

Storper M., Venables A.J. (2004) Buzz: Face-to-face contact and the urban economy. Journal of Economic Geography, vol. 4, pp. 351-370. Available at: https://doi.org/10.1093/jnlecg/lbh027, accessed 21.04.2019.

Warning S. (2007) The economic analysis of universities: Strategic groups and positioning, Cheltenham: Edward Elgar. 